\title{
ES Energy \& Environment
}

DOI: https://dx.doi.org/10.30919/esee8c260

\section{Micro/Nanoscale Thermal Characterization Based on Spectroscopy Techniques}

\author{
Jiaxin $\mathrm{Gu},{ }^{1}$ Jinjuan $\mathrm{She}^{2}$ and Yanan Yue ${ }^{1,2, *}$
}

\begin{abstract}
Low-dimensional materials, which possess extraordinary physical properties, bring huge opportunities in advanced thermal management. Upon their large-scale applications, understanding the thermal transport properties is essential. However, the micro-to-nanoscale diameter/thickness leads to extreme difficulties in thermal characterization. Few techniques have been developed in past years targeting specific structures. Regardless of how it works, accurate temperature probing and evaluation of heat spreading is most critical. Laser-based spectroscopy technique, such as micro-Raman, characterizes thermal properties based on temperature response of Raman scattering. The temperature probing from distinct peaks determines that the spatial resolution is not limited by the size of focused laser spot. Thus, it is capable of characterizing materials with dimension from macro down to nanoscale. This paper introduces the spectroscopy-based techniques developed in our lab for micro/nanoscale thermal characterizations. Raman as the primary one is detailed most. In addition, the advance of fluorescence spectroscopy techniques is briefly introduced. Due to the noncontact and non-destructive nature, the spectroscopy based technique has great potentials not only in micro/nanoscale characterization of thermosphysical properties, but also in extreme scale laser-assisted manufacturing field.
\end{abstract}

Keywords: Thermophyscial property; Micro/nanoscale; Raman; Fluorescence.

Received: 3 May 2020; Accepted date: 11 June 2020.

Article type: Review article.

\section{Introduction}

With miniaturization of electronic devices, the demand for heat dissipation is increased and proper thermal management becomes a crucial issue. Low-dimensional (LD) materials such as carbon nanotube (CNT) and graphene appear as ideal candidates due to their high thermal conductivity and small volume. ${ }^{[1]}$ Extensive work has demonstrated that thermal conductivity of monolayer graphene exceeds $1000 \mathrm{~W} / \mathrm{mK}$ at room temperature. ${ }^{[2-4]}$ However, its industrial applications are still limited by large variances in the thermal properties due to unpredictable defects. ${ }^{[5]}$ Besides, the accurate and consistent thermal characterization is another challenge, while it is also essential to the success of thermal design. ${ }^{[6]}$ The nanoscale geometry and fast transport properties largely impede the application of conventional techniques, such as hot disk method, laser flash, and so on. ${ }^{[7,8]}$

A few characterization techniques targeting low-

${ }^{1}$ School of Power and Mechanical Engineering, Wuhan University, Wuhan, Hubei 430072, China

${ }^{2}$ Department of Mechanical and Manufacturing Engineering, Miami University, Oxford, Ohio 45056, United States

*Email:yyue@whu.edu.cn (Y. Yue) dimensional and nanostructured materials have been developed in the past, and can be categorized as electrobased methods and optical methods depending on the way of heating and temperature sensing. ${ }^{[8-10]}$ In the meanwhile, the methods are also divided as steady-state and transient ones, based on the transport model used in the measurement. Frequently used electro methods are based on electrical heating or resistance thermal sensing. For example, the micro-bridge method, which is based on the steady-state resistance heating and temperature sensing of Pt wires, is capable of measuring thermal conductivity of micro/nanowires. ${ }^{[11]}$ For samples with effective laser absorption, the optical methods based on laser absorption or reflectance are a good option. ${ }^{[12]}$ They are mostly transient methods (such as laser reflectance), which usually involve photon heating and thermal probing. ${ }^{[13,14]}$ For $2 \mathrm{D}$ materials like graphene, not many optical methods are capable due to the ultra-thin thickness. Taking the laser reflectance method as an example, the temperature sensing is based on the reflectance of probing laser. The absorption of laser energy in the monolayer structure is limited. ${ }^{[15]}$ Laser penetrates the layer with little energy reflection. As such, the signal is either 


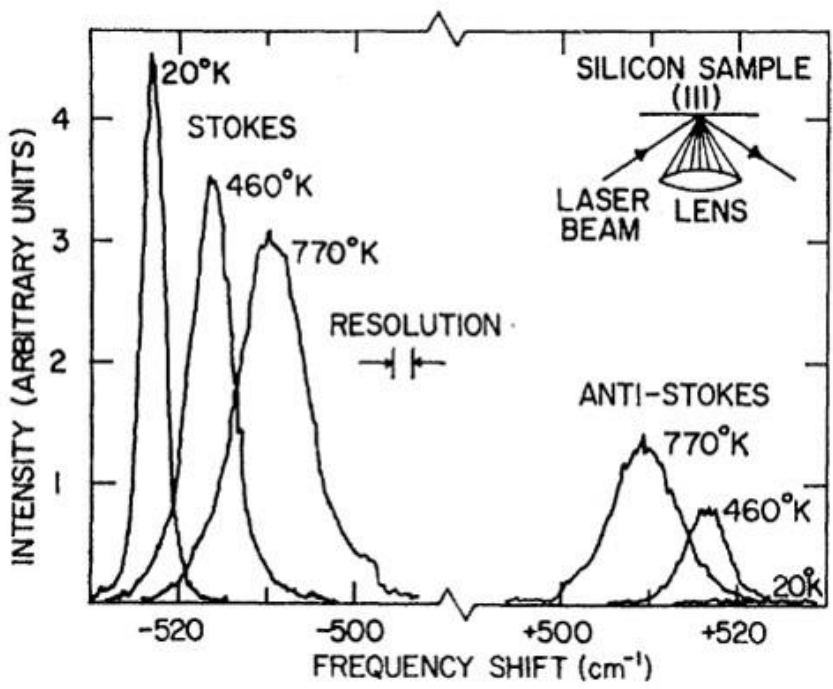

Fig. 1 Temperature dependence of Raman signal of silicon, reproduced with permission from [21].

too weak to be detected or what is detected is actually from other materials. ${ }^{[16]}$ Thus, the laser reflectance method cannot be directly implemented unless a certain treatment at surface is conducted. ${ }^{[17]}$ Other methods such as three omega method, transient electro-thermal method, have unique advantages in terms of characterizing thin films, microwires, or interface materials. ${ }^{[18-20]}$

Among various methods, Raman spectroscopy is very promising in terms of defining temperature based on scattering signal rather than the reflectance/radiation signal of materials. ${ }^{[21]}$ Raman is based on non-elastic scattering of materials when exposed with a continuous laser, showing as distinct peaks in the spectrum. ${ }^{[22,23]}$ As shown in Fig. 1 for Raman spectrum of silicon, the peak at certain photon frequency is temperature dependent, showing as peak shift (photon frequency), intensity and width (full width at half maximum, FWHM) change with temperature. ${ }^{[21]}$ These features can be used as the temperature indicator for thermal characterization, termed Raman thermometry. ${ }^{[24,25]}$ The Raman peak shifts to the lower-wave number direction as the temperature increases. Among different features to define temperature, the peak shift has higher sensitivity compared with other two features. ${ }^{[26]}$ The peak intensity is usually interfered by the focal level of laser beam during the measurement. The temperature dependence of peak width is not significant, but is a good reference because it is independent of strain effect. ${ }^{[16]}$ The selection of Raman features depends on the experimental conditions, like the quality of materials, resolution limit of spectrometer and the specification of the probing laser. ${ }^{[27]}$ For LD materials, even little dose of samples, e.g. monolayer graphene, individual CNT, the Raman signal (with a long time accumulation) is strong enough for analysis, which is instrumental for vast applications. ${ }^{[25,27]}$ High influential work employing Raman thermometry includes the first report of superior thermal 16| ES Energy Environ., 2020, 9, 15-27 conductivity of monolayer graphene ${ }^{[2]}$ and several work thereafter about the thermal conductivity measurement of graphene as a function of temperature and thermal conductivity measurement of monolayer $\mathrm{MoS}_{2}{ }^{\left[{ }^{[28,29]}\right.}$ To date, Raman spectroscopy has been accepted as one of the most powerful tools in micro/nanoscale thermal characterizations of LD materials. ${ }^{[30]}$

This paper introduces the spectroscopy-based techniques developed in our lab for micro/nanoscale thermal characterizations. Raman as the primary one is detailed most. In addition, the advance of fluorescence spectroscopy techniques is briefly introduced. The following content will be covered: Introduction of raman thermometry, Steady state Raman on 1D and 2D materials, Raman thermometry on 2D interface materials, Transient Raman thermometry, Tipenhanced Raman thermometry, and Advances in fluorescence thermometry.

\section{Steady state Raman on $1 D$ and $2 D$ materials}

\subsection{Joule heating technique on micro/nanowires}

The spatial resolution of Raman thermometry at nanoscale is not limited by the size of focused laser beam but by the size of the measurement target. For 1D structures, Raman can be applied based on either Joule heating or laser heating. ${ }^{[31]}$ The Joule heating method we developed is termed as "SERT" technique, standing for steady-state electroRaman-thermal technique. ${ }^{[24,32]}$ As shown in Fig. 2 for the measurement principle: a conductive micro/nanowire of length $2 L$ is suspended between two electrodes with its axial direction defined as the $\mathrm{x}$ direction and $\mathrm{x}=0$ is defined as the middle point of sample. The sample ends are assumed to be at room temperature during the measurement since they are attached on bulk metals (acting as electrode as well as the heat sink). During Joule heating, probing laser is focused on the middle to monitor temperature by Raman. The measurement should be conducted in a vacuum condition and protected with aluminum foil to avoid radiation heat loss. The middle temperature can be described as:

$$
T_{\mathrm{m}}=P \cdot L /\left(4 k \cdot A_{C}\right)+T_{\infty}
$$

where $P$ is heating power $\sim I^{2} R$, and $A c$ is the cross-sectional area of the sample. $k$ is thermal conductivity, which can be determined by the linear fitting of the relationship between $T_{\mathrm{m}}$ and $P$. Before the measurement, a calibration experiment is needed to establish the relationship between Raman signal and temperature. For very short samples (e.g. length smaller than laser spot size), the average temperature of the sample can be measured. In this way, the SERT technique is capable of measuring samples at nanoscale.

In thermal design of nanoelectronics, people consider not just heat conduction along the metallic wire, but also the heat convection effect from the wire to the surroundings. Under such circumstance, the heat convection effect should be considered, a Raman mapping technique can be used as

(C) Engineered Science Publisher LLC 2020 
shown in Fig. 3a: ${ }^{[33]}$ The heat dissipation of the wire is then described as:

$$
k A_{c} L \cdot d^{2} T / d x^{2}+Q-Q_{\text {air }}=0
$$

where $Q$ is heating power, $k$ is thermal conductivity, $A_{\mathrm{c}}$ is cross-sectional area, and $L$ is sample length. The term $Q_{\text {air }}$ stands for the convection heat loss: $h L S\left(T-T_{0}\right)$, where $h$ is convection coefficient, $S$ is wire perimeter, and $T_{0}$ is room temperature. Applying boundary condition that end points stay at room temperature, the temperature distribution is: ${ }^{[33]}$

$$
T(x)=\frac{Q}{h L S}\left(1-\frac{e^{\sqrt{\frac{h S}{k A}} x}+e^{-\sqrt{\frac{h S}{k A}} x}}{e^{\frac{\sqrt{\frac{h S}{k A}}}{2}}+e^{-\frac{\sqrt{\frac{h S}{k A}}}{2}}}\right)+T_{0}
$$

It shows that both $k$ and $h$ can be obtained by fitting temperature profile along the wire if the temperature profile along the sample is available.

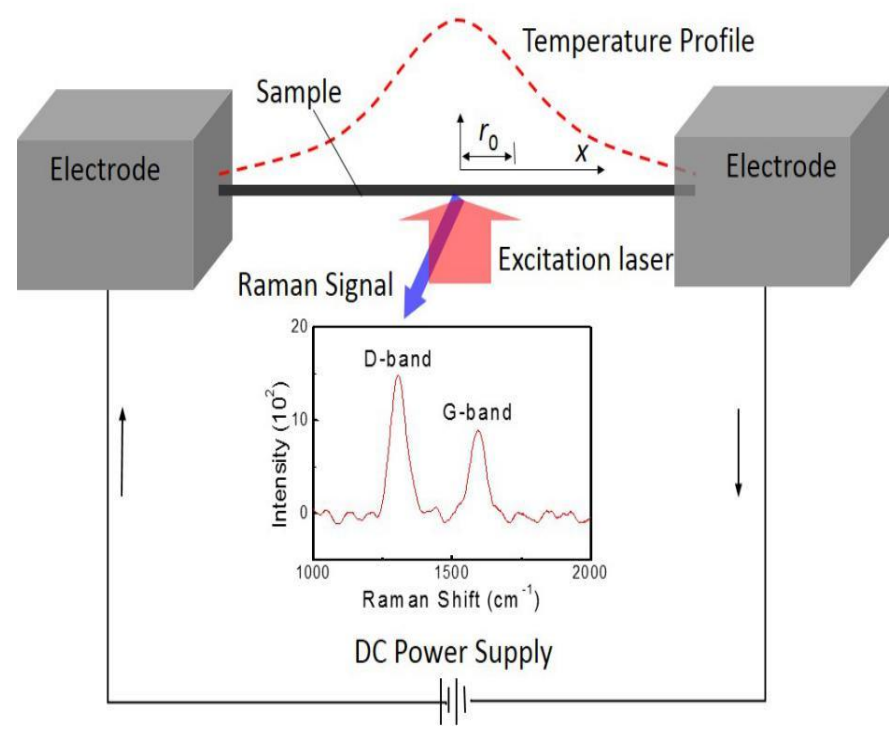

Fig. 2 Schematic of steady-state electro-Raman-thermal (SERT) technique, reproduced with permission from [32].

To validate this technique, a CNT fiber is selected for characterization. Five temperature points along the fiber $(\mathrm{x}=0$, $L / 10,2 L / 10,3 L / 10,4 L / 10)$ are characterized under different Joule powers. As shown in Fig. 3b, three peaks can be identified from 0 to $3000 \mathrm{~cm}^{-1}$ : the D-band $\sim 1350 \mathrm{~cm}^{-1}$, the G-band $\sim 1580 \mathrm{~cm}^{-1}$, and the 2D-band $\sim 2700 \mathrm{~cm}^{-1}$. The scanning electron microscopy (SEM) images display the morphology of the fiber. The high ratio of the length $(3.0 \mathrm{~mm})$ to the diameter $(36 \mu \mathrm{m})$ ensures one-dimensional thermal model applicable. ${ }^{[33]}$

The slope of 2D Raman peak with respect to temperature is obtained as $-0.050 \mathrm{~cm}^{-1} / \mathrm{K}$ (shown in Fig. 3c). Fig. $3 \mathrm{~d}$ illustrates the temperature distribution along the fiber. By fitting temperature profile using Eq. (1), both $k$ and $h$ are

$$
\bar{T}=\frac{1}{\pi r_{0}^{2}} \int_{0}^{2 \pi} d \varphi \int_{0}^{r_{0}} r T(r) d r=\frac{Q}{k}\left[\frac{1}{8 \pi d}+\frac{1}{2 \pi d}\left(\ln r_{L}-\ln r_{0}\right)\right]+T_{0}
$$

obtained at the same time in one temperature curve fitting. Thermal conductivity of CNTs fiber is obtained from 26 to $34 \mathrm{~W} / \mathrm{mK}$ for temperature from 335 to $468 \mathrm{~K}$ (as shown in Fig. 3e). The convection coefficient is characterized from 1143 to $1039 \mathrm{~W} / \mathrm{m}^{2} \mathrm{~K}$ monotonously for the same temperature range (shown in Fig.3f). ${ }^{[33]}$

\subsection{Laser heating method for 1D and 2D structure}

Heating effect from the probing laser is a concern but always ignored in most electrical methods. Somehow, such effect can be very significant if laser spot is extremely small and the excitation laser is powerful. In addition, if the sample is nonconductive but still Raman responsive, the laser heating method can be an option. The measurement principle is similar to SERT technique except that the heating source is switched to the laser spot heating. ${ }^{[34]}$ The governing equation for 1D steady-state model within laser irradiated region is $k \Delta T+\dot{q}=0$, where $T$ is temperature, $k$ is thermal conductivity, and $\dot{q}$ is equivalent heat generation rate $\left(\mathrm{W} / \mathrm{m}^{3}\right)$. $\dot{q}$ can be derived from the laser absorption $Q$ as: $Q / 2 \pi r_{s}^{2} r_{0}$, where $r_{\mathrm{s}}$ is sample radius, and $r_{0}$ is a half length of the laser beam. Outside laser heating region $\left(r>r_{0}\right)$, governing equation can be simplified as: $-k A \Delta T=Q / 2$, where $A$ is cross-sectional area of the sample. In laser heating region $\left(r<r_{0}\right)$, the temperature distribution is derived as:

$$
T(r)=-\frac{Q}{4 \pi k r_{0} r_{s}^{2}} r^{2}+\frac{Q}{2 \pi k r_{s}^{2}}\left(L-\frac{r_{0}}{2}\right)+T_{0}
$$

Thus, the average temperature in laser irradiation region can be derived as:

$$
\bar{T}=\frac{1}{r_{0}} \int_{0}^{r_{0}} T(r) d r=\frac{Q L}{2 \pi k r_{s}^{2}}-\frac{Q r_{0}}{3 \pi k r_{s}^{2}}+T_{0}
$$

The average temperature has a linear relationship with absorbed laser energy, and the coefficient can be used to calculate thermal conductivity.

2D materials feature extremely thin thickness and excellent crystalline structure, which is good for Raman excitation. Among 2D materials, graphene is studied most extensively. In Raman measurement, graphene is suspended with laser irradiated on the center. Heat dissipates from center to the edge. The local temperature can be monitored by Raman signals at different heating power levels. The governing equation in laser irradiated area is: $k$. $d[r d T / d r] / d r+r \cdot \dot{q}=0$, where $r$ is thermal transport radius, $k$ is thermal conductivity, and $\dot{q}$ is the equivalent heat generation rate (calculated as $Q / \pi r_{0}^{2} d$, where $r_{0}$ is laser radius, and $d$ is sample thickness). Outside heating area, the governing equation is $-k A d T / d r=Q$, where $A$ is crosssectional area of the sample. Applying boundary conditions, the average temperature in laser irradiation area is calculated by Equation (4): 
Since the center temperature is directly measured from Raman, thermal conductivity of 2D material can be obtained. This laser heating method can be applied on other thin films with size less than $1 \mathrm{um}$. In this case, the mean temperature can be described as: $\bar{T}=\bar{q} r_{L}^{2} / 8 k+T_{0}$, where $r_{L}$ is the effective radius of the sample.

Joule heating features accurate controlling of heat, but needs sample to be conductive. Since Raman signal is usually very weak, a certain level of laser power is needed to get sound signal. The measured temperature is not solely induced by Joule heating but a combined effect of Joule heating and laser heating. Although undesired heating effect can be eliminated by calibrating the system, very little inconsistency in focal levels between calibration and
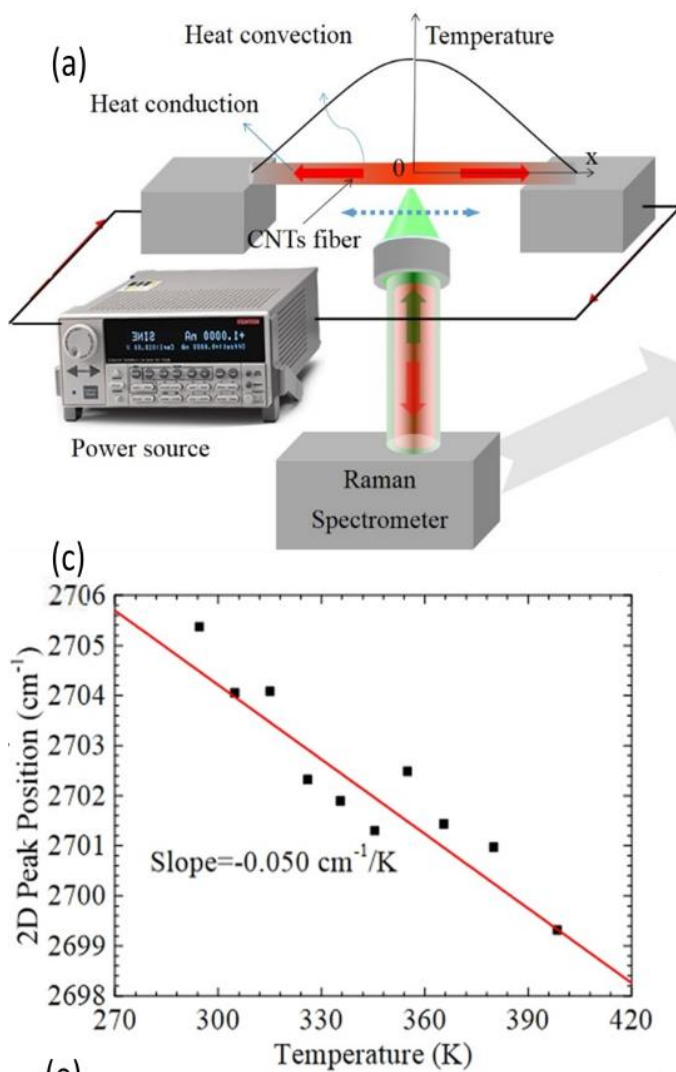

(e)

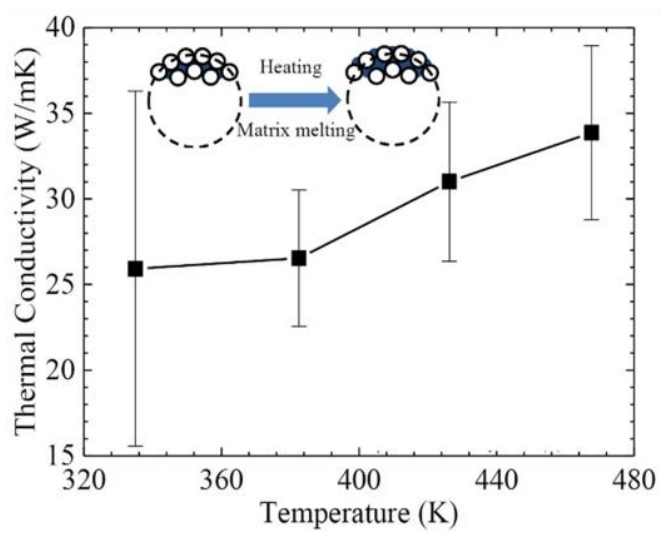

measurement could lead to large errors in temperature measurement. As to laser heating, the laser absorption should be precisely determined; otherwise, it would lead to direct errors in thermal conductivity measurement.

\section{Raman thermometry on 2D interface materials}

In applications, graphene is either supported by substrates or encased in dielectrics, where the interface thermal transport is important. Besides, it is reported that thermal conductivity of graphene is suppressed with the existence of substrate. ${ }^{[35]}$ It is very essential to characterize both interfacial thermal transport and in-plane thermal conductivity of supported 2D materials.
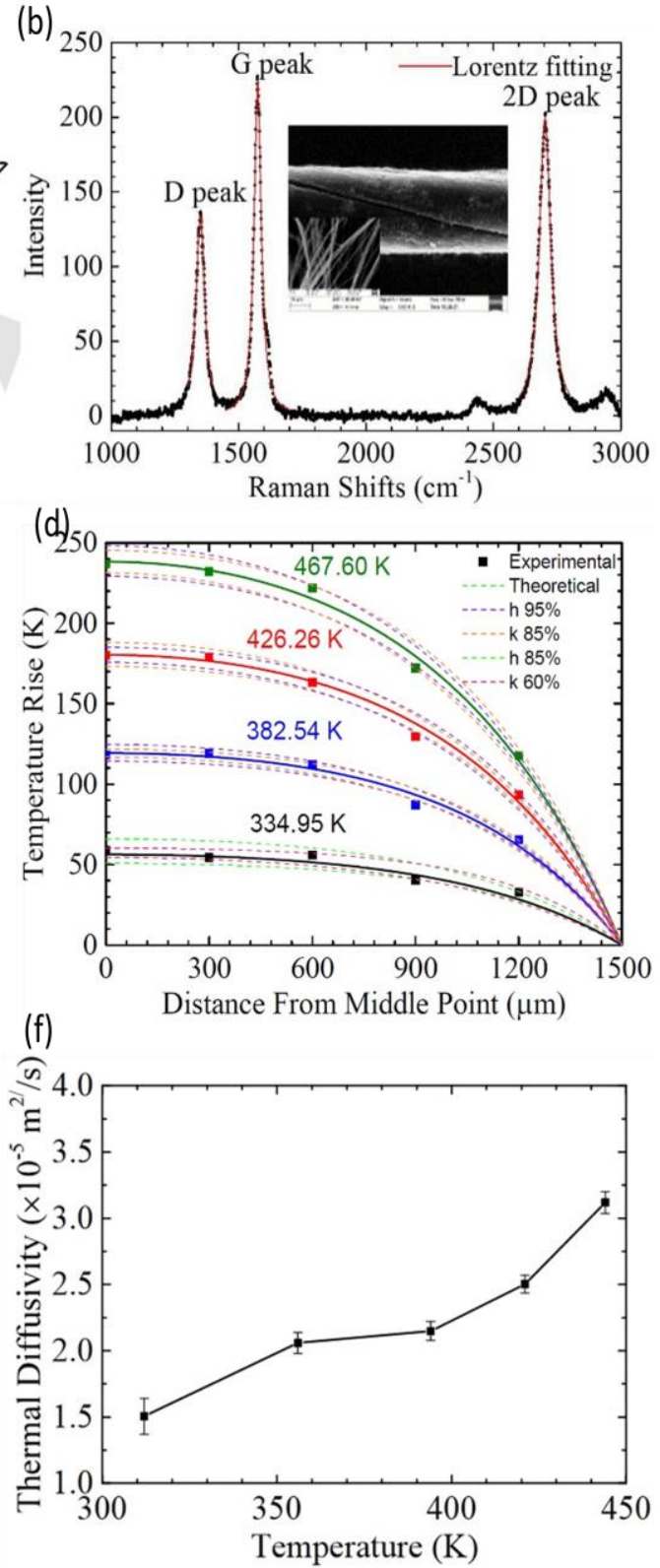

Fig. 3 (a) Schematic of Raman mapping technique; (b) Raman signal of CNT fiber; (c) calibration of Raman peak shift with respect to temperature; (d) temperature distribution along the fiber and the fitting result; (e) temperature dependence of thermal conductivity; (f) temperature dependence of thermal diffusivity, reproduced with permission [33]. 


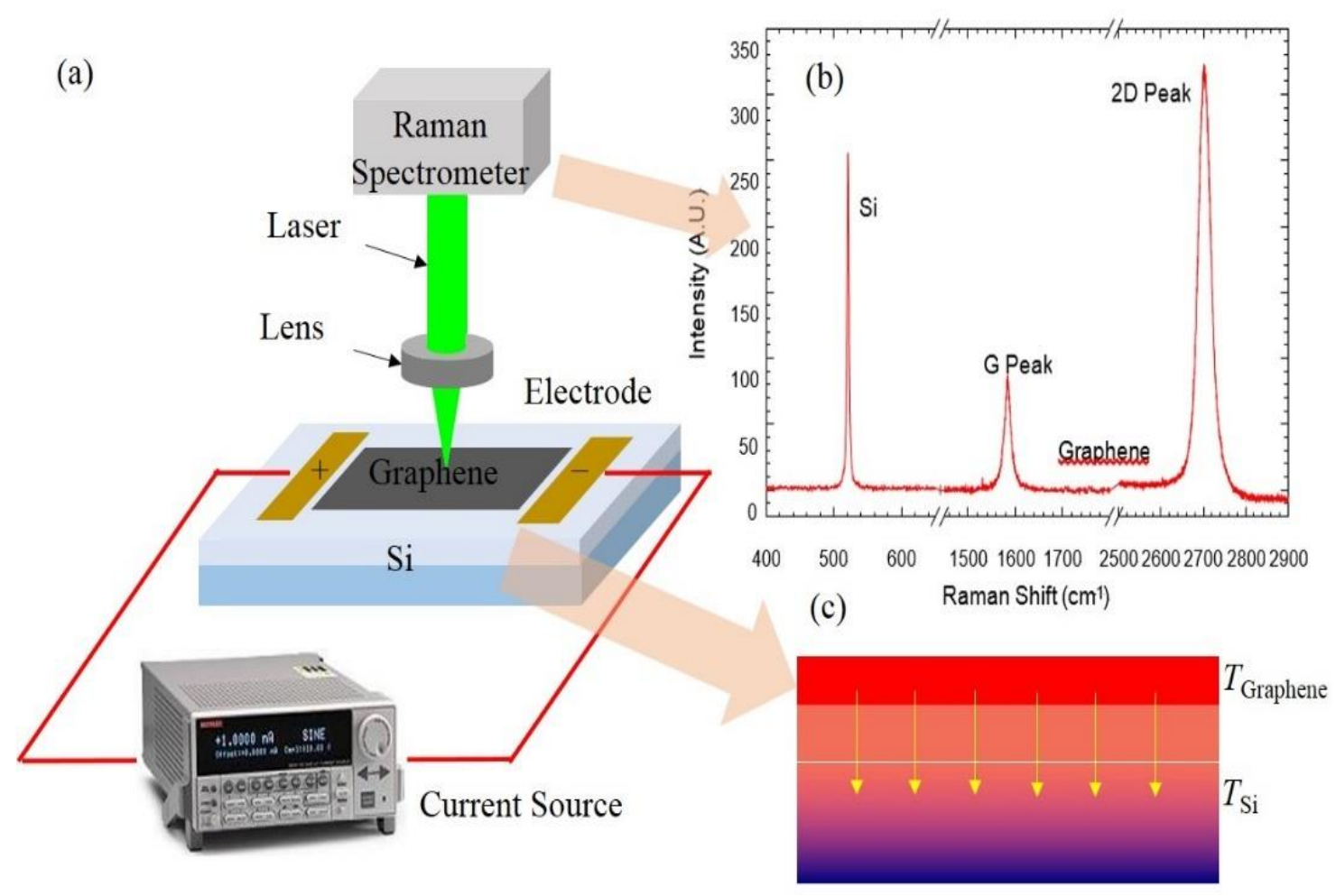

Fig. 4 (a) Schematic of Raman penetration method to characterize the interface thermal resistance between graphene and silicon; (b) Raman signal of graphene on silicon substrate; and (c) heat diffusion path from graphene to substrate, reproduced with permission from [36].

\subsection{Raman penetrating method for interface thermal resistance}

Raman can be used to characterize the interfacial thermal resistance between graphene and substrate based on electrical heating and Raman probing, which is termed as "Raman penetrating method". ${ }^{[15]}$ As shown in Fig. 4a, trimmed graphene flake is connected to current source for Joule heating. A constant current is supplied to generate uniform heating in graphene. The heat dissipates mainly through interface heat conduction to the substrate. Interfacial thermal resistance can be described as: $R=A \Delta T / Q$, where $Q$ is heating power, $\Delta \mathrm{T}$ is the temperature difference between graphene and substrate, and $A$ is interface area. Since graphene is very thin, Raman laser can easily penetrate it to reach the substrate. If the substrate is also Raman active (e.g. silicon), Raman signals of both graphene and substrate can be obtained in one spectrum, as shown in Fig. 4b: G peak $\sim 1580 \mathrm{~cm}^{-1}$ and 2D peak $2700 \mathrm{~cm}^{-1}$ for graphene, one signal at $\sim 520 \mathrm{~cm}^{-1}$ is from silicon. ${ }^{[22]}$ The temperature of graphene and silicon is obtained simultaneously and then used to calculate interface thermal resistance.

Prior to the application of Raman thermometry, a sound Raman signal for both graphene and silicon is required. A CVD synthesized graphene on silicon substrate is characterized for validating. Raman signals can be used to characterize the thickness (layer numbers) of graphene. As shown in Fig. 4b, the intensity ratio of G peak to $2 \mathrm{D}$ peak is much smaller than 1 , which indicates a monolayer structure of graphene. In the measurement, laser is focused on graphene with a relatively small power. The heat dissipates through the interface to the substrate, as shown in Fig. 4c. Note that Raman signal for monolayer structure could be very weak, a certain integration time is needed to get sound signals. The spectrum is averaged a few times to reduce measurement uncertainty. Various Joule powers are applied to graphene to get the relationship between temperature (Raman shift) and heating power for both graphene and silicon. Thus, the interfacial thermal resistance is obtained from $R_{g / S i}=A \cdot \Delta\left(T_{g}-T_{S i}\right) / \Delta Q$. In this Equation, the linear relationship between temperature and heating power is used to calculate thermal resistance with reduced uncertainties. In application scenarios, an oxidized layer of $\mathrm{SiO}_{2}$ on top of silicon always appears. Therefore, it needs to be cautioned that the measured value is composed of interfacial resistance between $\mathrm{Si}$ and $\mathrm{SiO}_{2}$, thermal resistance of $\mathrm{SiO}_{2}$ layer, and interfacial resistance between graphene and $\mathrm{SiO}_{2} \cdot{ }^{\left[{ }^{36]}\right.}$

\subsection{Two-step Raman method for in-plane thermal conductivity}

Most characterizations solely focus on either in-plane transport or cross-plane interfacial transport, while only few researchers consider the comprehensive transport for the interface materials. In electronic applications, the interface heat dissipation and in-plane thermal transport are coupled when a heat spot exists. Targeting this gap, a two-step 
approach based on Raman is developed for localized measurement of both thermal conductivity and interfacial thermal conductance. ${ }^{[36]}$ The two-step Raman involves the $1^{\text {st }}$ step on Joule heating Raman penetration method to characterize interfacial thermal resistance as introduced above, and the $2^{\text {nd }}$ step is about in-plane thermal conductivity measurement based on laser heating.

Different from the $1^{\text {st }}$ step that laser energy is maintained as low as possible, Raman laser in the $2^{\text {nd }}$ step should be powerful enough to result in significant temperature rise, which is affected not only by the interfacial thermal transport but also by the in-plane thermal conductance (as shown in Fig. 5a). Thermal transport in graphene is described as: ${ }^{[37]}$

$q=2 \pi k r_{1} \delta \theta m \frac{K_{1}\left(m r_{1}\right) I_{1}\left(m r_{2}\right)-K_{1}\left(m r_{2}\right) I_{1}\left(m r_{1}\right)}{K_{0}\left(m r_{1}\right) I_{1}\left(m r_{2}\right)+K_{1}\left(m r_{2}\right) I_{0}\left(m r_{1}\right)}$

where $q$ is the part of absorbed laser energy in 2D materials (graphene, as an example), which transfers in in-plane direction, $k$ is thermal conductivity of graphene, $\delta$ is thickness, $\theta$ is temperature rise of graphene, $m$ is defined as $m=\sqrt{2 h / k \delta}$ ( $h$ is interfacial thermal conductance between graphene and substrate), $r_{1}$ is the radius of heating region, $r_{2}$ is the radius of unheated part, and $I_{0}, K_{0}, I_{1}$, and $K_{1}$ are Bessel functions. ${ }^{[37]}$ In Eq. (5), $q$ can be obtained by subtracting the interface thermal transport calculated in the $1^{\text {st }}$ step. Therefore, thermal conductivity of graphene is the only unknown value in the equation and can be calculated accordingly.
In the measurement, only a small portion of laser energy is absorbed by graphene, which needs to be carefully treated. The absorptivity of monolayer graphene and $532 \mathrm{~nm}$ laser wavelength is only $2.3 \%$ from theoretical calculation. ${ }^{[38]}$ Little difference in laser absorption would result in large difference in thermal conductivity. For graphene $/ \mathrm{SiO}_{2} / \mathrm{Si}$ interface as shown in Fig. 5b, there are multiple laser transmissions and reflections. If all are counted, the total absorption of laser energy would be $3.2 \%$ of total laser energy. In order to reduce uncertainty in the measurement, Eq. (5) is derived as:

$$
\frac{d \theta}{d q}=\frac{1}{2 \pi k r_{1} \delta m} \times \frac{K_{0}\left(m r_{1}\right) I_{1}\left(m r_{2}\right)+K_{1}\left(m r_{2}\right) I_{0}\left(m r_{1}\right)}{K_{1}\left(m r_{1}\right) I_{1}\left(m r_{2}\right)-K_{1}\left(m r_{2}\right) I_{1}\left(m r_{1}\right)}
$$

As shown in Fig. 5c, the temperature coefficient of graphene against laser power is fitted as $5.40_{-1.5}^{+2.1} \times 10^{6} \mathrm{~K} / \mathrm{W}$ In the measurement, the temperature of silicon is monitored to see if it remains at room temperature during laser heating. With other parameters being applied, the relationship between interfacial thermal conductance and thermal conductivity can be calculated, as shown in Fig. 5d. Thermal conductivity is obtained as $179_{-86}^{+111} \mathrm{~W} / \mathrm{mK}$, which is a low value comparing to suspended structure. It might be caused by phonons leakage and scattering with the existence of substrate. ${ }^{[36]}$

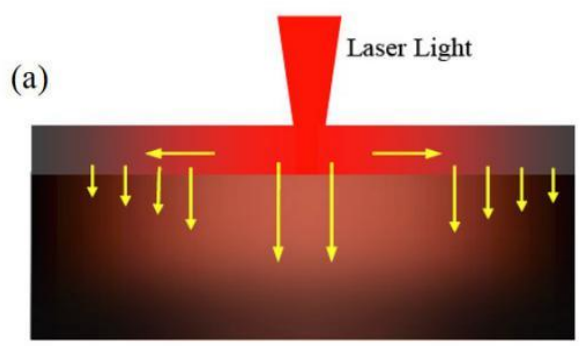

(c)

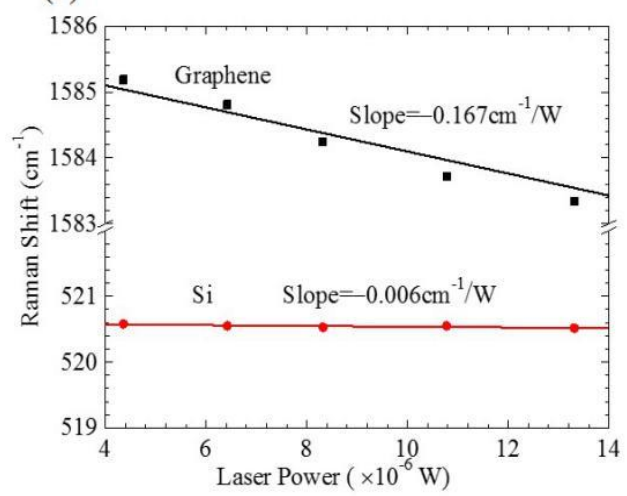

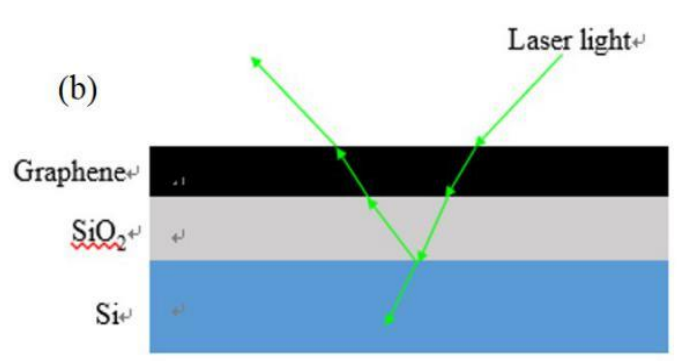

(d)

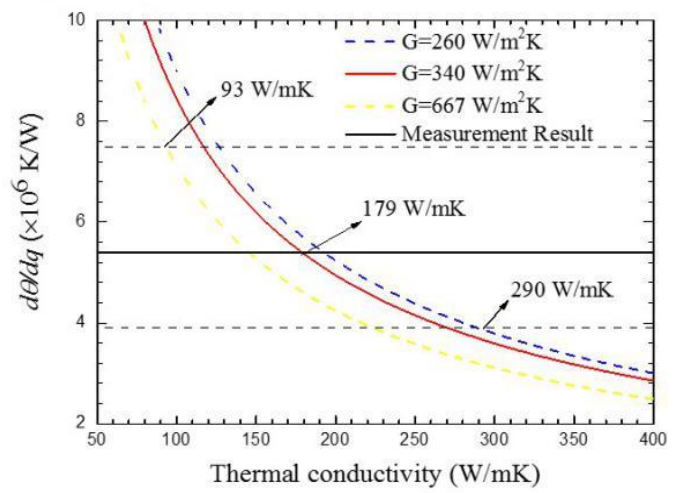

Fig. 5 (a) Thermal transport under laser spot heating; (b) laser reflection and refraction when $\mathrm{SiO}_{2}$ layer exists; (c) Raman shift of graphene and silicon with respect to laser heating power; and (d) data processing for deriving thermal conductivity, reproduced with permission from [36]. 

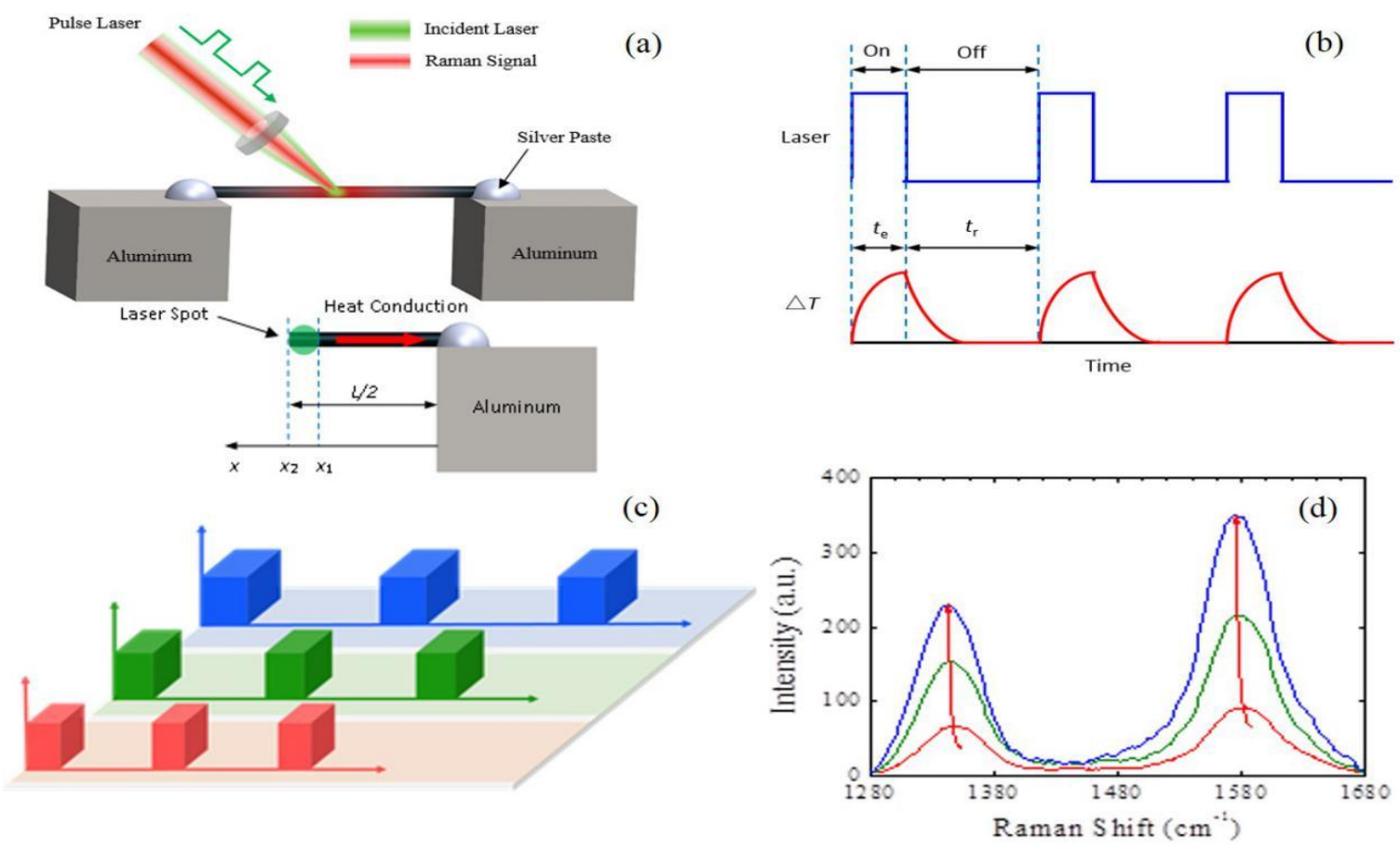

Fig. 6 (a) Schematic of time-domain Raman method for 1D thermal characterization; (b) temperature response under pulse laser heating; (c) examples of laser modulation and on-off duration setting; and (d) result of Raman shift under different laser pulse heating in (c), reproduced with permission [39].

\section{Transient Raman thermometry}

Conventional Raman thermometry is steady-state since Raman is excited from a continuous laser. It is desirable to develop a comprehensive Raman method, which is also capable of transient measurement. Time-domain differential Raman (TD-Raman) is developed under such demand. ${ }^{[39]}$

\subsection{Physical model for TD-Raman}

Similar to previous setup, as shown in Fig. 6a, the sample is suspended between heat sinks for 1D characterization. A continuous laser is modulated to generate square-wave pulse with variable laser-on/off durations. As shown in Fig. 6b, the laser beam consists of an excitation period $\left(t_{\mathrm{e}}\right)$ followed by a relaxation period $\left(t_{\mathrm{r}}\right)$. In excitation period, the sample is heated and Raman scattering is collected. Raman signal here is an integration value from signals at various temperatures over laser heating period. Thermal relaxation period should be long enough to allow the sample fully cool down before the next cycle. Sufficient "heating and cooling" cycles are repeated (by adjusting integration time) to obtain Raman spectrum. In case that laser spot is larger than sample diameter and the length-to-diameter ratio is also large enough, the 1D thermal transport model is suitable for laser heating experiment: $\rho c_{p} \partial \theta / \partial t=k \partial^{2} \theta / \partial x^{2}+q_{0}$, where $\rho$ and $c_{p}$ are density and specific heat; $\theta$ is temperature rise, $k$ is thermal conductivity, and $q_{0}$ is the heat generation per unit volume from laser. Considering the symmetry, the spatially averaged temperature rise in the heated region can be expressed as:

$$
\bar{\theta}(t)=\frac{2 q_{0} L^{3}}{k\left(x_{2}-x_{1}\right)} \sum_{m=1}^{\infty} \frac{1}{m^{4} \pi^{4}}\left[1-\exp \left(-\frac{m^{2} \pi^{2} \alpha t}{L^{2}}\right)\right]\left(\cos \frac{m \pi}{L} x_{1}-\cos \frac{m \pi}{L} x_{2}\right)^{2}
$$

where $\alpha$ is thermal diffusivity of the sample and $L$ is the sample length. The coordinates of $x_{1}$ and $x_{2}$ are shown in Fig. $6 \mathrm{c}$. During the heating period $\left(t_{\mathrm{e}}\right)$, average temperature rise in the heated region can be obtained from the accumulated Raman signal. Thermal diffusivity of the sample can be fitted from the normalized temperature rise against heating time as: 


$$
\bar{\theta}^{*}=\frac{\bar{\theta}(t)}{\bar{\theta}(t \rightarrow \infty)}=\frac{\sum_{m=1}^{\infty} \frac{1}{m^{4} \pi^{4}}\left[1-\exp \left(-\frac{m^{2} \pi^{2} \alpha t}{L^{2}}\right)\right]\left(\cos \frac{m \pi}{L} x_{1}-\cos \frac{m \pi}{L} x_{2}\right)^{2}}{\sum_{m=1}^{\infty} \frac{1}{m^{4} \pi^{4}}\left(\cos \frac{m \pi}{L} x_{1}-\cos \frac{m \pi}{L} x_{2}\right)^{2}}
$$
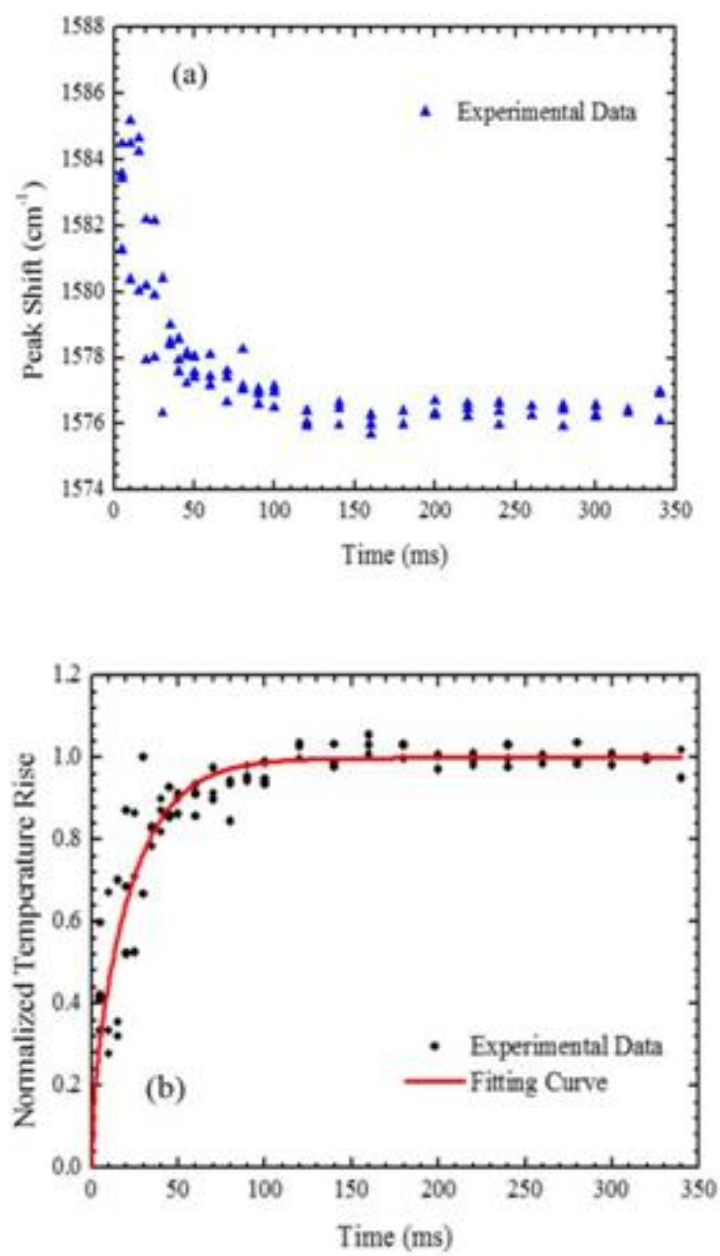

Fig. 7 (a) Experimental result of Raman shift changes with respect to laser heating duration; and (b) fitting result of normalized temperature rise with respect to heating duration, reproduced with permission [39].

\subsection{Experimental demonstration}

A CNT fiber is employed to demonstrate the effectiveness of this technique. Fig. $6 \mathrm{~d}$ shows three selected Raman spectra of the CNT fiber sample under different excitation time $\left(t_{\mathrm{e}}\right)$ in one "heating and cooling" cycle. With the increase of excitation time, Raman peaks (D band and G band) shift to the shorter wavenumber direction. This shift is attributed to the temperature rise in the heating period with $t_{\mathrm{e}}$ increases. Raman intensity is also increased because of longer excitation time. The continuous laser is modulated to generate variable square-wave pulse laser. The duty ratio: $t_{\mathrm{e}} /\left(t_{\mathrm{e}}+t_{\mathrm{r}}\right)$ of the square-wave pulse laser is set to be $5 \%$ in the measurements. The heating time in one cycle is adjusted from 5 to $340 \mathrm{~ms}$ by switching modulation frequency, e.g. 10 $\mathrm{Hz}$ for $5 \mathrm{~ms}$ heating ( $t_{\mathrm{e}}=$ duty/frequency). The characteristic $22 \mid$ ES Energy Environ., 2020, 9, 15-27 time of heat transport along the sample can be estimated as $0.81 l^{2} / \alpha,{ }^{[19,40]}$ where $l$ is half length of the sample. With a referenced thermal diffusivity of $1.51 \times 10^{-5} \mathrm{~m}^{2} / \mathrm{s}$, ${ }^{[33]}$ the characteristic time of thermal transport in this fiber is 53.6 $\mathrm{ms}$. The shortest relaxation time for the case of $5 \mathrm{~ms}$ heating experiments is $95 \mathrm{~ms}\left(t_{\mathrm{r}}\right)$, which ensures that the sample has no heat accumulation

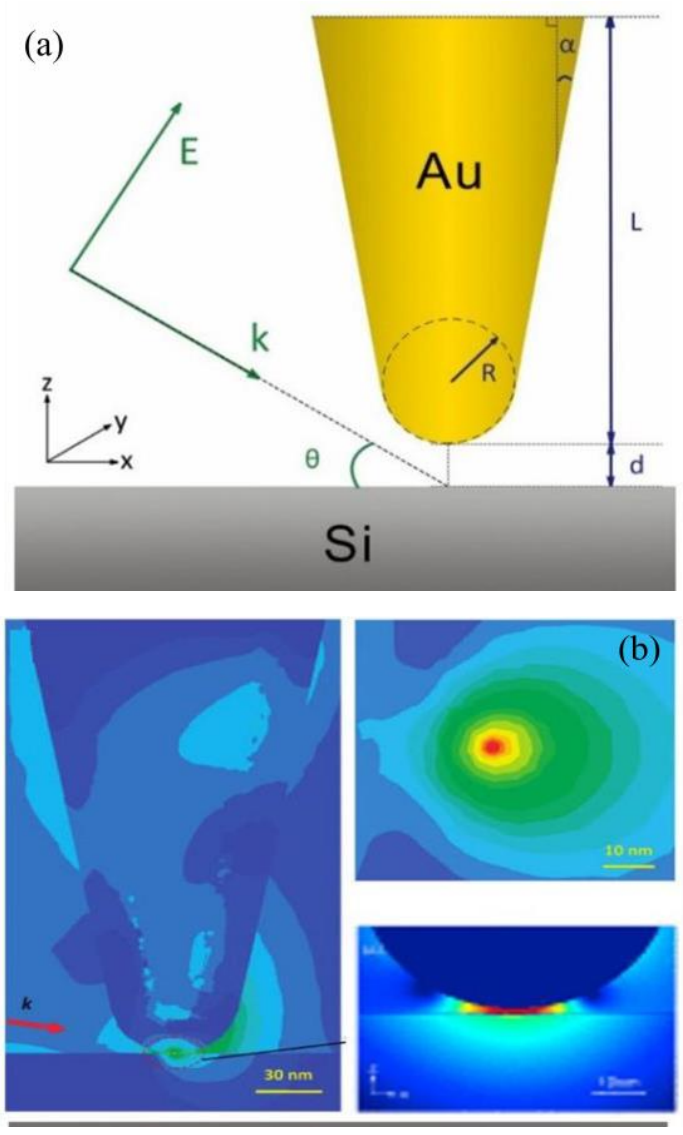

Fig. 8 (a) A Schematic of AFM tip-substrate configuration for generation near field effect [42] (b) simulation results of tipinduced optical enhancement, ${ }^{[42,43]}$ reproduced with permission.

Fig. 7a illustrates measurement results when sample is heated by square-wave laser energy. During excitation, the average temperature is measured using $\mathrm{G}$ band peak shift. The G-band peak shift decreases quickly at the beginning of the laser heating time, indicating that temperature increases rapidly before heat transfers to the electrodes. While heating time is increased, the shift variation slows down toward a constant value as the temperature of sample reaches steady state. Based on the good linearity between peak shift and temperature, the normalized temperature rise of the CNT fiber is obtained from: $\bar{\theta}^{*}=\left[\omega(t)-\omega_{0}\right] /\left(\omega_{s}-\omega_{0}\right)$, where

(C) Engineered Science Publisher LLC 2020 


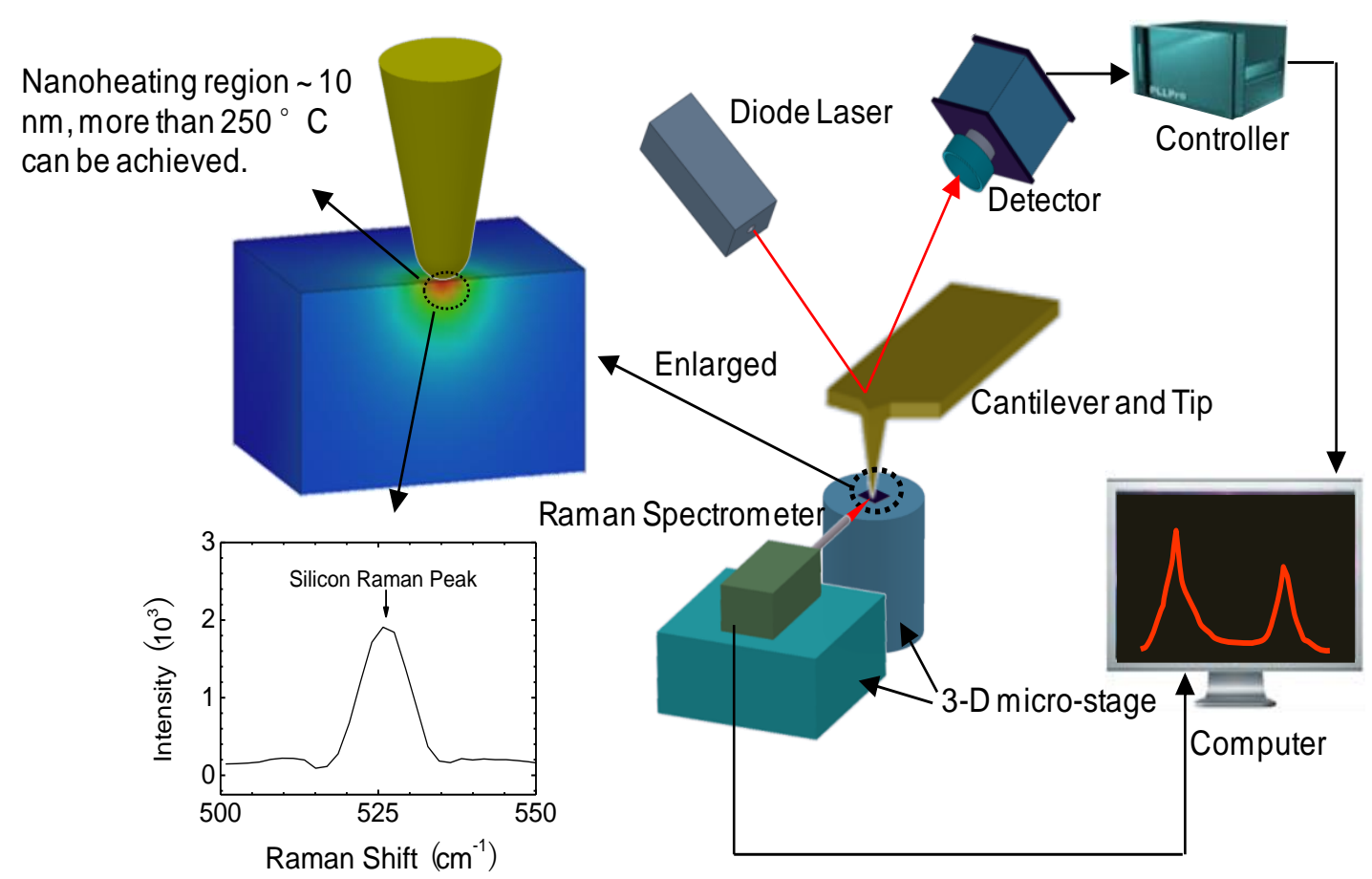

Fig. 9 Experimental setup for tip-enhanced Raman thermometry, reproduced with permission from [43].

where $\omega(t)$ is the peak shift at different heating time, $\omega_{0}$ and $\omega_{s}$ are the peak shift of the sample measured at room temperature and steady-state, respectively. Fig. $7 \mathrm{~b}$ shows the relationship between normalized temperature increase and heating time. Thermal diffusivity is calculated as $1.74_{-0.20}^{+0.20} \times$ $10^{-5} \mathrm{~m}^{2} / \mathrm{s}$ by using least square fitting with Eq. (8). ${ }^{[39]}$

\section{Tip-enhances Raman thermometry}

\subsection{Diffraction limit and near field effect}

The nanoscale hot spot become a critical issue in electronics.

The spatial resolution of conventional optical thermometry such as inferred thermometer is limited by the intrinsic diffraction. Therefore, the resolution limit is only in the order of light wavelength. One approach to break the diffractionlimit barrier is to place the excitation source or detection probe close to the sample surface with a distance less than the wavelength. This is named as near-field scanning optical microscopy (NSOM), which has the capacity to deal with the phenomenon smaller than $100 \mathrm{~nm} \cdot{ }^{[41]}$ The AFM tip is an ideal tool to produce the near-field effect. Fig. 8 shows a simulation example of using tip to generate near field effect within a confined region. ${ }^{[42]}$ In the configuration, an external laser is irradiated on the side or the bottom of the tip. Enhanced optical field appears in a small region beneath the tip. Such optical enhancement can be used to improve Raman scattering rate during the laser-matter interaction, known as tip-enhanced Raman. Since Raman signals can be easily obtained due to the optical enhancement beneath the tip and thus it can be used to characterize the temperature at nanoscale.

\subsection{Tip-enhanced Raman thermometry}

A typical schematic for tip-enhanced Raman thermometry is shown in Fig. 9. Raman spectrometer is placed in front of the AFM with the laser focusing on the tip. The silicon substrate is employed to validate this effect and placed beneath the Au-coated silicon tip, where the near-field heating is generated because of the optical field enhancement. Measurement of the substrate temperature using Raman requires an established relationship between temperature and the Raman spectra. The calibration of Raman signal of silicon with respect to temperature is conducted in advance. In the measurement, to ensure temperature is from the silicon substrate rather than the tip, the AFM tip (made of silicon) is coated with a certain thickness gold.

In the measurement, the AFM is operated in contact mode (very close to the substrate). A silicon substrate is placed on a 3-D micro-stage under the tip. The laser beam passes through a beam splitter and a focal lens to irradiate on the tip apex to generate near field heating and enhances Raman signal. Spectrometer is placed in front of the tip to detect Raman signal. If possible, the spectrometer and laser could be integrated together for convenient adjustment. To prevent signals directly from unheated region of the silicon, the 

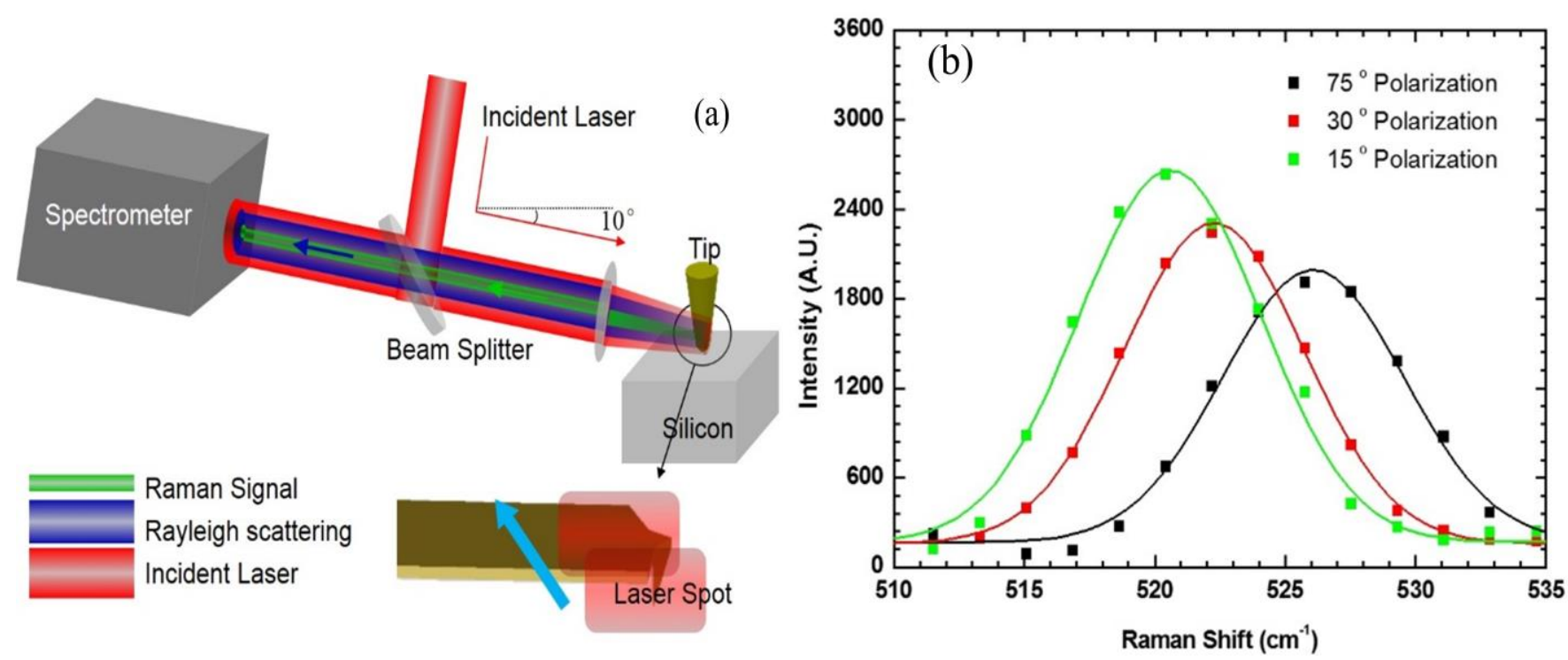

Fig. 10 (a) Optical alignment of laser-tip interactions; (b) measurement result under different polarization angles, reproduced with permission from [43].

incident angle of the laser beam is kept very small $\left(80^{\circ}\right.$ with respect to the tip axis). Three different polarization angles $\left(15^{\circ}, 30^{\circ}\right.$, and $\left.75^{\circ}\right)$ are used for checking the effect of the polarization on the substrate heating. As shown in Fig. 10a for the optical alignment, the incident laser passes through a beam splitter and is focused on the tip by a convex lens. The focal point of the laser beam is large than the tip. Thus, the scattered Rayleigh signal, which provides an effective way to monitor the focal position.

Results for three polarization angles are shown in Fig. 10b. It can be seen that temperature (Raman shift) for the $15^{\circ}$ and $30^{\circ}$ polarization angles is significant. For $75^{\circ}$ polarization angle, the temperature increase is very marginal, which confirms well with theoretical analysis. ${ }^{[43]}$ Based on this, the nanoscale thermal probing has been achieved.

\section{Advances in fluorescence thermometry}

Besides Raman, fluorescence is another spectroscopy technique which advances stronger signal and shorter accumulation time for capturing enough photon energy. It is an ideal tool for temperature sensing and then thermal characterization if the measurement target is fluorescence active. ${ }^{[25]}$ The size-dependent band gap from quantum confinement and edge effects of nanoscale materials (like quantum dot) enables the excitation of fluorescence signals..$^{[44}$

\subsection{Principle of fluorescent thermometry}

The temperature dependence of fluorescence spectroscopy has been studied extensively. ${ }^{[45,46]}$ Fig. 11a shows the temperature dependence of fluorescence spectrum of graphene quantum dots (GQDs) ${ }^{[44]}$ It shows that the intensity decreases with the increase of temperature. Fig. $11 \mathrm{~b}$ presents the temperature dependence of normalized fluorescence intensity for different wavelengths, showing that there is a significant decrease within a small temperature range. ${ }^{[4]}$

The fluorescence intensity can be obtained from $F_{I}=\Phi$. $I \cdot B$, where $\Phi$ is quantum efficiency, a temperaturedependent parameter according to the Boltzmann distribution: $A \cdot \exp (-E / k T)$, where $A$ is a constant, $E$ is state energy, $k$ is the Boltzmann's constant and $T$ is mperature. It shows that the quantum efficiency as well as the corresponding fluorescence intensity decreases when temperature is increased. The red shift of fluorescence signal is another feature for temperature sensing. Sometimes, the fluorescence spectrum of materials (e.g. GQD) is broad and the red shifts phenomenon is not apparent. Compared with peak intensity and shift, the peak width is not as sensitive as the other two. ${ }^{[44]}$

\subsection{Steady-state Fluorescence}

Based on fluorescence thermometry, the steady-state electrical-heating fluorescence-sensing (SEF) technique is developed. ${ }^{[47]}$ This method shares the same experimental setup with SERT technique. ${ }^{[24]}$ If a sample itself is a good fluorescence dye, the signal from the sample surface can be used for temperature measurement. If the size of a sample is larger than the laser spot, the laser spot is focused on the middle of the sample during measurement. For a sample without fluorescence excitations, quantum dots (QDs) can be used as fluorescence dye for thermal probing. ${ }^{[25,47]}$ In this case, QDs are coated on the sample surface and the measured fluorescence signal represents local temperature of the sample. ${ }^{[47]}$

\subsection{Transient fluorescence}

(c) Engineered Science Publisher LLC 2020 
SEF technique is capable of measuring thermal conductivity of materials, and it can be combined with a transient fluorescence spectroscopy to study thermal diffusivity. Referring to the TD-Raman method, fluorescence signal is collected during laser heating period to define temperature. ${ }^{[39,40,48]}$ If heating pulse is very short compared to heat diffusion time of the sample (especially for polymers with extremely low thermal conductivity), the thermal transport is only confined within a very thin layer near the sample surface. A semi-infinite model is applicable to describe the temperature evolution during laser heating, and the temperature rise at sample surface can be derived as:

$T(t)=\frac{2 q_{0} \sqrt{a t / p}}{k} \exp \left(-x^{2} / 4 a t\right)-\frac{q_{0} x}{k} \operatorname{erfc}(x / 2 \sqrt{a t})+T_{0}$
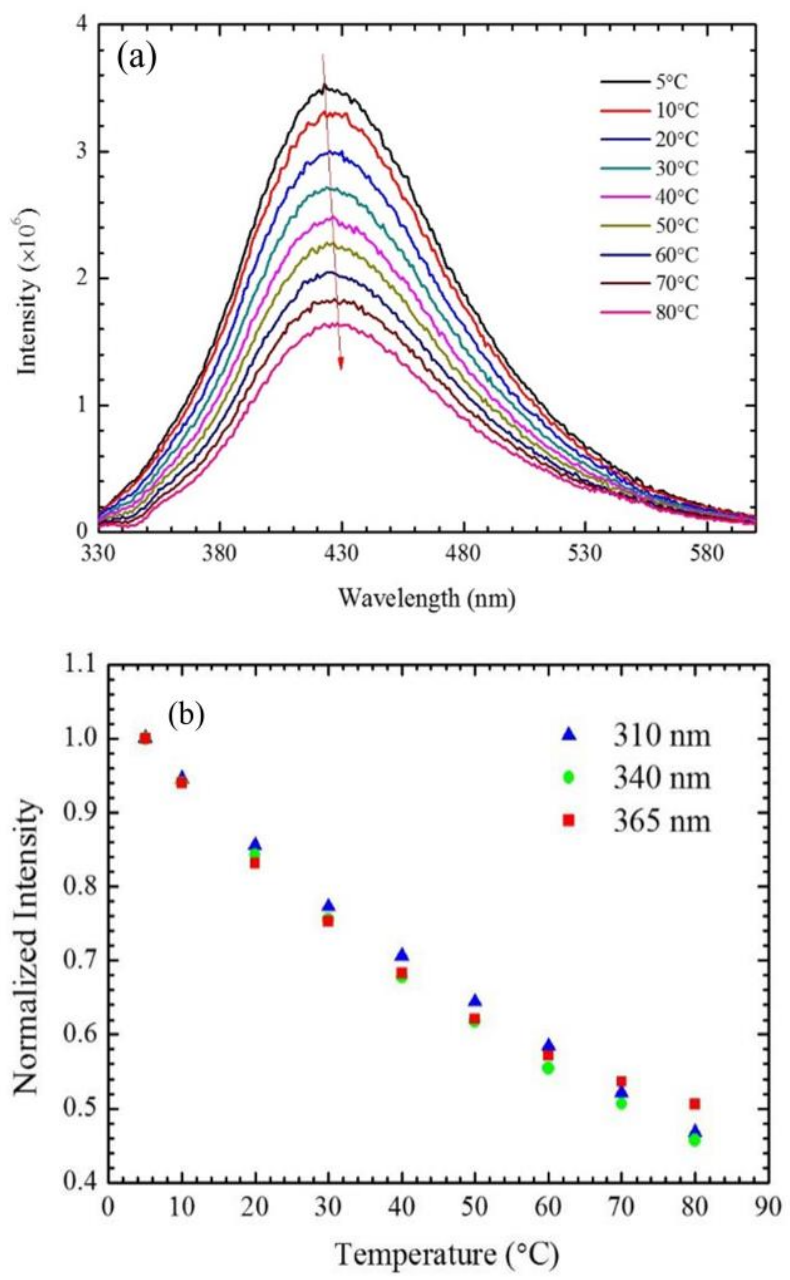

Fig. 11 (a) Fluorescence signal of GQDs under different temperature; (b) normalized fluorescence intensity with respect to temperature, reproduced with permission [44]. where $\alpha$ is thermal diffusivity, $k$ is thermal conductivity, $q_{0}$ is heat flux density induced by laser heating, $T_{0}$ is room temperature, and erfc is error function. It is shown that $\alpha$ can be obtained from temperature rise against heating time.

In measurement, the heat source $q_{0}$ (laser absorption) is yet hard to be characterized, especially for unknown or new materials. To avoid such uncertainty, a normalized temperature rise $T(t)^{*}=\left[T(t)-T_{0}\right] /\left(T_{m}-T_{0}\right)$ is defined, where $T_{0}$ is room temperature and $T_{\mathrm{m}}$ is the maximum temperature rise. Meanwhile, the temperature coefficient is not necessary for thermal diffusivity measurement. By defining:

$$
F(t)=2 \sqrt{\frac{\alpha t}{\pi}} \exp \left(-\frac{x^{2}}{4 \alpha t}\right)-x \cdot \operatorname{erfc}\left(\frac{x}{2 \sqrt{\alpha t}}\right)
$$

The normalized temperature rise is derived as:

$$
T(t)^{*}=\left[t_{m} \cdot \int_{0}^{x} \int_{0}^{t} F(t)\right] /\left[t \cdot \int_{0}^{x} \int_{0}^{t_{m}} F(t)\right],
$$

where $t_{m}$ is the maximum heating time. $\alpha$ can be obtained from the normalized temperature rise against heating time.

To test this model, a standard polymer (poly vinyl chloride, PVC) with known properties is employed for characterization. PVC is one of widely produced synthetic polymers with strong fluorescence signal. Its thermal conductivity is around 0.16 $\mathrm{W} / \mathrm{m} \cdot \mathrm{K}$ from literatures. ${ }^{[49]}$ By applying this technique, thermal conductivity is characterized as $0.19 \mathrm{~W} / \mathrm{m} \cdot \mathrm{K}$, aligning roughly well with reference data considering the sample differences. Thermal diffusivity is obtained as $1.031 \times 10^{-7} \mathrm{~m}^{2} / \mathrm{s}$, also in good agreement with reported values characterized by other techniques. ${ }^{[50]}$ In the measurement, it is required to minimize the quenching effect, which leads to the intensity decrease caused by various factors. The laser energy should be set as a minimum value to avoid too much heat accumulation. In addition, the heating period is carefully controlled to be short enough to reduce quenching. ${ }^{[48]}$

Aside from the fluorescence intensity, the lifetime of fluorescence is also temperature dependent and is a promising field to explore in the future. By employing a single-photon counter, the lifetime-based fluorescence thermometry would achieve high temporal resolution characterization. ${ }^{[51]}$

\section{7. Conclusions and perspectives}

Laser spectroscopy techniques are growing fast in micro/nanoscale thermal characterizations. In the meantime, the limitation of measurement capacity applies. Raman thermometry is constrained to Raman active materials, and mostly narrowed to some crystalline semiconductors. Fluorescence has broader applications considering the fact 
that semiconductor QDs can be used as additive materials to excite signal. However, as to extreme measurement such as 2D materials, Raman is an irreplaceable tool. Besides laser spectroscopy techniques, other methods like pump-probe and micro-bridge methods are also very successful and are capable of characterizing micro/nanoscale structures in lots of scenarios.

This review narrows the characterization methods to the laser-spectroscopy ones, rather than the electrical based methods and other laser-based ones. In general, the optical based methods are limited by the diffraction effect, the resolution of which is usually at the level of laser wavelength, except for the near-field based techniques. The accuracy of the temperature measurement for spectroscopy based methods can be diverse. For Raman based methods, the accuracy is limited by the spectral resolution of the Raman spectrometer since the peak shift is always employed as the temperature indicator. Typical gratings used for Raman vary from $300 \mathrm{gr} / \mathrm{mm}$ to $1800 \mathrm{gr} / \mathrm{mm}$ which give a temperature accuracy roughly from $10 \mathrm{~K}$ to $1 \mathrm{~K}$. But if more specialized gratings (such as $2400 \mathrm{gr} / \mathrm{mm}$ and $3600 \mathrm{gr} / \mathrm{mm}$ ) are equipped, the accuracy would be improved significantly. Not to mention the peak fitting process, which would improve the accuracy further to below $1 \mathrm{~K}$ easily. In fluorescence thermometry, the peak intensity is used for defining temperature rather than the peak shift. Its accuracy is highly dependent on the sensitivity of fluorescence signal of measurement target, which varies material to material. The advantages of spectroscopy based method are distinct: (1) They are optical based methods which are noncontact and nondestructive. (2) The signals are directly from the measurement target and thus the characterization of nanoscale features would be feasible. The most challenging part of using these techniques is the collection of spectroscopy signals as well as the analyzing process. Taking Raman as an example, the signal is usually not strong for nanoscale structures and a long integration time is needed for collecting a sound signal. But as to thermal characterization, the heating process would ruin the sample if it is not treated properly. Therefore, the selection of proper method is very essential to the successful thermal characterizations at micro/nanoscale.

\section{Acknowledgements}

Authors acknowledge the support from the National Natural Science Foundation of China (No. 52076156 and 51576145).

\section{Conflict of Interest}

There are no conflicts to declare.

\section{Support information}

Not applicable

\section{References}

[1] M. Fujii, X. Zhang, H. Xie, H. Ago, K. Takahashi, T. Ikuta, H. Abe and T. Shimizu, Phys. Rev. Lett., 2005, 95(6), 065502, doi: 10.1103/PhysRevLett.95.065502.

[2] A. Balandin, S. Ghosh, W. Bao, I. Calizo, D. Teweldebrhan, F. Miao and C. N. Lau, Nano Lett.,2008, 8(3), 902-907, doi: 10.1021/n10731872.

[3] S. Ghosh, I. Calizo, D. Teweldebrhan, E. Pokatilov, D. L. Nika, A. A. Balandin, W. Bao, F. Miao and C. N. Lau, Appl. Phys. Lett., 2008, 92(15), 151911, doi: 10.1063/1.2907977.

[4] J. Hu, X.Ruan and Y. P. Chen, Nano Lett., 2009, 9(7), 2730-2735, doi: 10.1021/n1901231s.

[5] C. Liu, M. Chen, W.Yu and Y. He, ES Energy Environ., 2018, 2, 31-42, doi: 10.30919/esee8c191.

[6] H. Zhang, C. Hua, D. Ding and A. J. Minnich, Sci. Rep., 2015, 5(1), 9121, doi: 10.1038/srep09121.

[7] D. G. Cahill, P. V. Braun, G. Chen, D. R. Clarke, S. Fan, K. E. Goodson, P.Keblinski, W. P. King, G. D. Mahan, A. Majumdar, H. J. Maris, S. R. Phillpot, E. Pop and L. Shi, Appl. Phys. Rev., 2014, 1(1), 011305, doi: 10.1063/1.4832615.

[8] D. G. Cahill, W. K. Ford, K. E. Goodson, G. D. Mahan, A. Majumdar, H. J. Maris, R.Merlin and S. R.Phillpot, J. Appl. Phys., 2003, 93(2), 793-818, doi: 10.1063/1.1524305.

[9] D. G. Cahill, P. V. Braun, G. Chen, D. R. Clarke, S. Fan, K. E. Goodson, P. Keblinski, W. P. King, G. D. Mahan, A. Majumdar, H. J. Maris, S. R. Phillpot, E. Pop and L. Shi, Appl. Phys. Rev., 2014, 1(1), 011305, doi: 10.1063/1.4832615.

[10] W. Ma, T. Miao, X. Zhang, K. Takahashi, T. Ikuta, B. Zhang and Z. Ge, Nanoscale, 2016, 8(5), 2704-2710, doi: 10.1039/c5nr05946a.

[11] P. Kim, L. Shi, A. Majumdar and P. L. McEuen, Phys. Rev. Lett., 2001, $\quad \mathbf{8 7 ( 2 1 ) ,} \quad 215502, \quad$ doi: 10.1103/PhysRevLett.87.215502.

[12] H. Zhang, X. Chen, Y. D. Jho and A. J. Minnich, Nano Lett., 2016, $\quad \mathbf{1 6 ( 3 )}, \quad$ 1643-1649, doi: 10.1021/acs.nanolett.5b04499.

[13] A. J. Schmidt, X. Chen and G. Chen, Rev. Sci. Instrum., 2008, 79(11), 114902, doi: 10.1063/1.3006335.

[14] B. Guo, J. Sun, Y. Lu and L. Jiang, Inter. J. Extreme Manufac., 2019, 1(3), 032004, doi: 10.1088/2631-7990/ab3a24.

[15] Y. Yue, J.Zhang and X. Wang, Small, 2011, 7(23), 33243333, doi: 10.1002/smll.201101598.

[16] Y. Yue, J. Zhang, Y. Xie, W. Chen and X. Wang, Int. J. Heat Mass Tran., 2017, 110, 827-844, doi: 10.1016/j.ijheatmasstransfer.2017.03.082.

[17] P. E. Hopkins, M. Baraket, E. V. Barnat, T. E. Beechem, S. 
P. Kearney, J. C. Duda, J. T. Robinson and S. G. Walton, Nano Lett., 2012, 12(2), 590-595, doi: 10.1021/nl203060j.

[18] J. H. Kim, A. Feldman and D. Novotny, J. Appl. Phys., 86(7), 3959-3963, doi: 10.1063/1.371314.

[19] J. Guo, X. Wang and T. Wang, J. Appl. Phys., 2007, 101(6), 063537, doi: 10.1063/1.2714679.

[20] C. Dames, Annual Revi. Heat Transfer, 2013, 16, 7-49, doi: 10.1615/AnnualRevHeatTransfer.v16.20.

[21] T. Hart, R. Aggarwal and B. Lax, Phys. Rev. B,1970, 1(2), 638-642, doi: 10.1103/PhysRevB.1.638.

[22] L. Malard, M.Pimenta, G. Dresselhaus and M. Dresselhaus, Phys. Rep., 2009, 473(5-6), 51-87, doi: 10.1016/j.physrep.2009.02.003.

[23] B. Michael, C. Kristian, W. Ferdinand, B. Martin, De L. Dominique and S. Michael, Inter. J. Extreme Manufac., 2020, 2, 3, doi: 10.1088/2631-7990/ab9583.

[24] Y. Yue, G. Eres, X. Wang and L. Guo, Appl. Phys. A,2009, 97(1), 19-23, doi: 10.1007/s00339-009-5352-6.

[25] Y. Yue and X. Wang, Nano Rev.,2012, 3(1), 11586, doi: 10.3402/nano.v3i0.11586.

[26] Y. Yue, J. Zhang, X. Tang, S. Xu and X. Wang, Nanotechnol. Rev.,2015, 4(6), 533-555, doi: 10.1515/ntrev-2014-0024.

[27] M. Dresselhaus, G. Dresselhaus, R. Saito and A. Jorio, Phys. Rep., 2005, 409(2), 47-99, doi: 10.1016/j.physrep.2004.10.006.

[28] J. U. Lee, D. Yoon, H. Kim, S. W. Lee and H. Cheong, Phys. Rev. B, 2011, 83(8), 081419, doi: 10.1103/PhysRevB.83.081419.

[29] R. Yan, J. R. Simpson, S. Bertolazzi, J. Brivio, M. Watson, X. Wu, A. Kis, T. Luo, A. R. Hight Walker and H. G. Xing, ACS Nano, 2014, 8(1), 986-993, doi: 10.1021/nn405826k.

[30] Q. Y. Li, W. G. Ma and X. Zhang, Int. J. Heat Mass Tran., 2016, 95, 956-963,

10.1016/j.ijheatmasstransfer.2015.12.065.

[31] J. Liu, T. Li, Y. Hu and X. Zhang, Nanoscale, 2017, 9(4), 1496-1501, doi: 10.1039/c6nr06901k.

[32] Y. Yue, X. Huang and X. Wang, Phys. Lett. A, 2010, 374(40), 4144-4151, doi: 10.1016/j.physleta.2010.08.034.

[33] M. Li, C. Li, J. Wang, X. Xiao and Y. Yue, Appl. Phys. Lett., 2015, 106(25), 4923189, doi: 10.1063/1.4923189.

[34] M. Li and Y. Yue, J. Nanosci. Nanotechno., 2015, 15(4), 3004-3010, doi: 10.1166/jnn.2015.9641.

[35] J. H. Seol, I. Jo, A. L. Moore, L. Lindsay, Z. H. Aitken, M. T. Pettes, X. Li, Z. Yao, R. Huang, D. Broido, N. Mingo, R. S. Ruoff and L. Shi, Science, 2010, 328(5975), 213-216, doi: 10.1126/science. 1184014 .

[36] W. Zhao, W. Chen, Y. Yue and S. Wu, Appl. Therm. Eng., 2017, 113, 481-489, doi: 10.1016/j.applthermaleng.2016.11.063. [37] T. L. Bergman, F. P. Incropera, D. P. DeWitt and A. S. Lavine, Fundamentals of heat and mass transfer: John Wiley\& Sons, 2011.

[38] R. R. Nair, P. Blake, A. N. Grigorenko, K. S. Novoselov, T. J. Booth, T. Stauber, N. M. Peres and A. K. Geim, Science, 2008, 320(5881), 1308, doi: 10.1126/science.1156965.

[39] C. Li, S. Xu, Y. Yue, B. Yang and X. Wang, Carbon, 2016, 103, 101-108, doi: 10.1016/j.carbon.2016.03.003.

[40] S. Xu, T. Wang, D. Hurley, Y. Yue and X. Wang, Opt. Express, 2015, 23, 10040-10056, doi: 10.1364/OE.23.010040.

[41] R. C. Dunn, Chem. Rev., 1999, 99(10), 2891-2927, doi: $10.1021 / \mathrm{cr} 980130 \mathrm{e}$.

[42] Y. Chen, Y. Xu, D.Xie, J. Jiang and Y. Yue, Appl. Therm. Eng., 2019, 148, 129-135, doi: 10.1016/j.applthermaleng.2018.11.050.

[43] Y. Yue, X. Chen and X. Wang, ACS Nano, 2011, 5(6), 4466-4475, doi: 10.1021/nn2011442.

[44] C. Li and Y. Yue, Nanotechnology, 2014, 25(43), 435703, doi: 10.1088/0957-4484/25/43/435703.

[45] V. Biju, Y. Makita, A. Sonoda, H. Yokoyama, Y. Baba and M. Ishikawa, J. Phys. Chem. B, 2005, 109(29), 13899-13905, doi: 10.1021/jp0504241.

[46] S. A. Wade, S. F. Collins and G. W. Baxter, J. Appl. Phys., 2003, 94(8), 4743-4756, doi: 10.1063/1.1606526.

[47] X. Wan, C. Li, Y. Yue, D. Xie, M. Xue and N. Hu, Nanotechnology, 2016, 27(44), 445706, doi: 10.1088/09574484/27/44/445706.

[48] H. Wu, K. Cai, H. Zeng, W. Zhao, D. Xie, Y. Yue, Y. Xiong and X. Zhang, Appl. Therm. Eng., 2018, 138, 403-408, doi: 10.1016/j.applthermaleng.2018.04.076.

[49] C. Choy, Polymer,1977, 18(10), 984-1004, doi: 10.1016/0032-3861(77)90002-7.

[50] W. N. Dos Santos, P. Mummery and A. Wallwork, Polym. Test., 2005, 24(5), 628-634, doi: 10.1016/j.polymertesting.2005.03.007.

[51] Y. Yue, H. Wu and Y. Xiong. (China, 2020), chap. CN 107907517A, pp. CN 107907517A.

Publisher's Note: Engineered Science Publisher remains neutral with regard to jurisdictional claims in published maps and institutional affiliation. 